\title{
Paroxysmal tinnitus due to a meningioma in the cerebellopontine angle
}

\author{
Michael Espir, Robin Illingworth, Borka Ceranic, Linda Luxon
}

39 Hyde Park Gardens Mews, London

W2 2NX, UK

M Espir

Department of

Neurosciences,

Charing Cross

Hospital, Fulham

Palace Road, London

W6 8RF, UK

R D Illingworth

Institute of

Laryngology and

Otology, 330-332

Gray's Inn Road,

London WC1X 8EE,

UK

L M Luxon

B Ceranic

Correspondence to:

Dr Espir.

Received 22 February 1996 and in revised form

11 November 1996

Accepted 3 December 1996

\begin{abstract}
A 44 year old woman presented with frequent paroxysms of unilateral tinnitus and ataxia which were abolished by treatment with carbamazepine. Hearing was normal and initially there were no other symptoms or signs between paroxysms. Brain stem evoked responses from the affected left ear were absent and MRI showed a large tumour in the left cerebellopontine angle. This was completely removed and histologically proved to be a meningioma. There were striking similarities to trigeminal neuralgia and other paroxysmal brain stem symptoms which occasionally occur in multiple sclerosis and diseases of the cerebellopontine angle. Possible mechanisms are discussed.
\end{abstract}

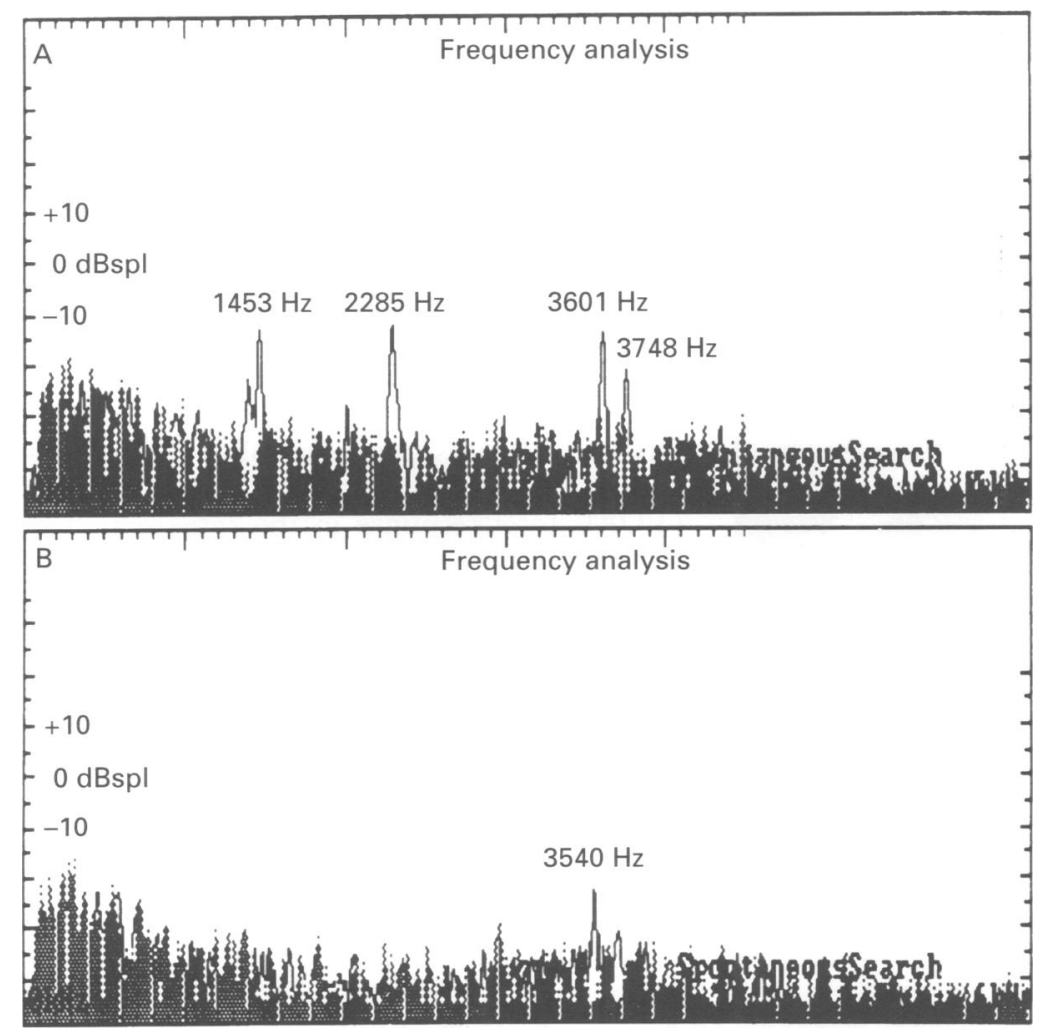

Figure 1 Frequency spectrum of the spontaneous otoacoustic emissions recorded in the left ear; (A) preoperatively, showing distinct peaks at $1453 \mathrm{~Hz}, 2285 \mathrm{~Hz}, 3601 \mathrm{~Hz}$, and $3748 \mathrm{~Hz}$, and (B) postoperatively, showing almost complete cessation of emissions.
$(\Im$ Neurol Neurosurg Psychiatry 1997;62:401-403)

Keywords: meningioma; cerebellopontine angle; tinnitus

Unilateral subjective tinnitus is a common presenting symptom of cerebellopontine angle tumours; it may be persistent or intermittent, and occasionally occurs without hearing loss. ${ }^{1}$ We describe here a patient who had a meningioma in the cerebellopontine angle and presented with the unusual symptom of paroxysmal tinnitus.

\section{Case report}

A 44 year old married woman working as a secretary had a two and a half month history of intermittent episodes of a noise "like the screech of brakes of an underground train" in her left ear. This lasted for 5-10 seconds and recurred several times each hour without any obvious provocation. With each episode she also felt unsteady as though she had "just got off a boat", and on a few occasions she had had to sit down on the ground. In between these attacks she felt entirely normal. She had no hearing loss.

About three years previously for two weeks she had felt unsteady "like being on a boat all the time". About a year previously she had had continuous tinnitus in the left ear which cleared after about six months. She had also had recurrent bouts of stiffness in her neck during the past year. She had received treatment with prochlorperazine and a cervical collar without any benefit. She had no other neurological symptoms and no other relevant medical or family history.

On examination during the episodes she was slightly unsteady but there was no nystagmus. Her hearing was clinically normal; the left corneal reflex was normal when she was seen first but later was slightly reduced; there were no other neurological signs.

Pure tone audiometry, speech reception thresholds, and tympanometry were normal. Ipsilateral and contralateral reflexes were absent on stimulating the left ear, but normal on the right. Transient click evoked otoacoustic emissions (TEOAEs) were present in both ears, indicating normal cochlear func- 
tion, with a stronger total response of 13.0 dBspl in the left ear, and 9.3 dBspl in the right. Spontaneous otoacoustic emissions (SOAEs) were recorded in the left ear only, with four spectral peaks at $1453 \mathrm{~Hz}, 2285 \mathrm{~Hz}$, $3601 \mathrm{~Hz}$, and $3748 \mathrm{~Hz}$ (fig $1 \mathrm{~A}$ ).

Contralateral sound mediated suppression of the emissions was reduced bilaterally. Brain stem auditory evoked responses (AERs) showed no reproducible responses on stimulating the left ear, neither ipsilaterally nor contralaterally, and were normal on the right. Standard electro-oculographic battery assessing gaze evoked nystagmus, optokinetic responses, saccades and pursuit were normal, except for saccadic intrusions of pursuit attributable to carbamazepine. Caloric responses showed a left canal paresis of $35 \%$.

Brain MRI showed a $3 \times 2 \mathrm{~cm}$ mass in the left cerebellopontine angle extending into the internal auditory meatus which was not expanded. The left side of the pons was very compressed and indented. The mass was rather flat and bun-like in shape and for this reason it was thought that it might be a meningioma rather than a schwannoma (fig 2).

Within 24 hours of starting treatment with carbamazepine (tegretol retard; $200 \mathrm{mg}$ twice daily) the paroxysms of tinnitus and ataxia were abolished.

At operation on 9 June 1994 via a posterior fossa craniectomy and drilling open the internal auditory meatus, the tumour was completely removed including the part which had extended into the meatus. Histology showed a benign mesothelial and psammomatous meningioma.

Her postoperative course was uneventful; there was no deafness or other neurological deficit apart from slight ataxia and a feeling of vibration intermittently in the left ear. Treatment with carbamazepine was not continued after the operation and the tinnitus did not recur.

Figure 2 Brain MRI showing tumour in the left cerebellopontine angle.
On repeating the tests of eighth nerve function postoperatively, there was no significant change in the pure tone audiogram. Acoustic reflex responses and BSERs from the left ear were not reproducible but the canal paresis documented on caloric testing had increased to $58 \%$. Pursuit was normal after stopping carbamazepine. The SOAE from the left ear had almost entirely ceased (fig 1B).

\section{Discussion}

Unilateral tinnitus, hearing loss, and unsteadiness, with an absent corneal reflex are common presenting features of cerebellopontine pathology, neuroma arising on the vestibular division of the eighth nerve being the commonest. ${ }^{2}$ In $25 \%$ of cases, other diseases are identified, meningiomas being the most common (about $8 \%$ of cases). ${ }^{34}$ The normal hearing and the MRI findings in our patient suggested a meningioma ${ }^{4}$ rather than an acoustic neuroma.

Tinnitus may be due to lesions of the labyrinth, eighth nerve, or CNS, but the pathophysiology remains obscure. Hypotheses include decoupling of the stereocilia of the hair cells, "misinterpretation" of auditory neural activity by higher centres, self sustaining oscillation of the basilar membrane consequent on the evoked cochlear mechanical response ${ }^{5}$ and alterations in the neural activity within the eighth cranial nerve.$^{67}$ As early as 1944, fibre interaction in injured or compressed neural tissue was reported $^{8}$ and one theory of tinnitus generation proposed by Moller $^{9}$ is that damage to the myelin sheath may allow ephaptic transmission ("crosstalk") between nerve fibres, which results in derangement of the temporal pattern of auditory nerve discharges and gives rise to tinnitus. Another less well documented mechanism for the development of tinnitus is the derangement of the efferent auditory fibres, which run in the vestibular division of the eighth nerve, producing aberrant auditory behaviour. ${ }^{10}$ In this case, tinnitus was associated with an increased spontaneous discharge rate in the auditory nerve, similar to the mechanism suggested by Moller ${ }^{9}$ and supported by the fact that the tinnitus was abolished by carbamazepine. The presence of SOAEs, and higher TEOAE total response in the same ear, may indicate increased cochlear output on that side as a result of dysfunction of the central cochlear control mechanisms. However, in the absence of information regarding the previous cochlear state, this remains speculative.

It is of interest that a year before presentation, this patient reported tinnitus which was continuous for about six months without hearing loss, and which then remitted. This may have been consequent on progressive auditory nerve damage as a result of pressure, which produced initially minor damage and altered neural activity within the eighth nerve perceived as tinnitus. ${ }^{9}$ With increasing pressure, degeneration of these affected fibres resulted in resolution of the tinnitus, or there may have been damage to the efferent system as mani- 
fest by the reduction of contralateral sound mediated suppression of otoacoustic emissions, ${ }^{11}$ and thus a reduction in the "damping" of spontaneous mechanical activity within the Organ of Corti or production of abnormal afferent auditory activity, ${ }^{10}$ giving rise to tinnitus. Alternatively with increase in size of the tumour and increasing pressure, neural degeneration of the affected afferent fibres responsible for transmitting disordered neural activity from the cochlea may have improved the perception of tinnitus. A further explanation for resolution of the continuous tinnitus may be related to plasticity within the $\mathrm{CNS},{ }^{6}$ facilitating adaptation to the disordered input as may occur with respect to vestibular pathology in lesions of the cerebellopontine angle lesions. Indeed, vertigo is not a prominent symptom in such lesions because of central compensation with a gradual loss of vestibular function.

Paroxysmal vertigo and ataxia are recognised in some peripheral and central vestibular syndromes. However, the combination of paroxysmal tinnitus and ataxia as in this patient has not been reported previously and a number of hypotheses should be considered.

It is recognised that the root entry zone of cranial nerves is particularly sensitive to mechanical pressure..$^{912}$ Moreover, the auditory nerve is covered by central myelin for 15-20 $\mathrm{mm}^{13}$ compared with only a few $\mathrm{mm}$ for the fifth and seventh cranial nerves. Moller ${ }^{9}$ concluded that the eighth nerve is therefore particularly vulnerable to pressure changes, although most of the literature on this mechanism is concerned with cases of hemifacial spasm and trigeminal neuralgia related to vascular anomalies ${ }^{12}$ and tumours ${ }^{314}$ involving the seventh and fifth nerve respectively. More recently Moller et al ${ }^{15}$ have reported that some patients with severe tinnitus have vascular compression of the auditory portion of the eighth cranial nerve close to the nerve's entrance to the brain stem and that microvascular decompression can alleviate the tinnitus in about $40 \%$ of such patients.

A case of paroxysmal tinnitus and synchronous nystagmus, accompanied by facial spasm in association with a vascular loop, has been reported by Isu et $a l^{16}$ whereas Kondo et al ${ }^{17}$ have reported that eight of 13 patients with hemifacial spasm had "paroxysmal" tinnitus. Bertrand et al $^{14}$ reported intermittent but not paroxysmal tinnitus in one of five patients with episodic vestibular syndromes resulting from vascular loops or arachnoid adhesions in the cerebellopontine angle, but in these patients the dysequilibrium lasted hours rather than seconds. Jannetta ${ }^{18}$ identified one patient with paroxysmal vertigo but no auditory symptoms in a large series of 117 patients with trigeminal neuralgia, hemifacial spasm, acoustic nerve dysfunction and glossopharyngeal neuralgia presumed secondary to compression at the root entry or exit zone of the relevant nerve as a result of arterial loops, tumours, veins or, very rarely, some other structural abnormality.

Various paroxysmal brain stem disturbances have been recorded during the course of multiple sclerosis $^{19}$ and may be the presenting symptom. ${ }^{20}$ The most popular theory for the pathogenesis of the paroxysmal symptoms in multiple sclerosis is of spreading ephaptic activation of axons within a partially demyelinated lesion. However, in our patient there was no evidence of multiple sclerosis and postoperatively she recovered entirely. All forms of paroxysmal symptoms are usually controlled by carbamazepine and the typically dramatic response of the paroxysms in our patient seems consistent with the postulate that the drug acts by depressing synaptic and ephaptic transmission and axonal excitability.

We thank Mr H B Holden for referring the patient, Mr Stewart Anderson for preoperative neurophysiological testing, Dr Paul Lewis for the histological report, Mr A D Cheesman for his contribution to the operation, the Photographic Unit at the contribution to the operation, the Photographic Unit at the
Institute of Laryngology and Otology, the Royal National Institute of Laryngology and Otology, the Royal National
Throat, Nose and Ear Hospital for fig 1, and $\mathrm{Mr}$ Tom Crawford for fig 2 .

1 Luxon LM. Tinnitus: its causes, diagnosis and treatment. BMF 1993;306:1490-1.

2 Brackmann DE, Kwartler JA. 1983-1988. A review of acoustic tumours. Am f Otolaryngol 1990;11:216-32.

3 Revilla AG. Differential diagnosis of tumours at the cerebellopontine recess. Fohns Hopkins Hospital Bulletin 1948; 83:187-203.

4 Prasher D, Coelho A, Nadkarni J, Luxon LM. Differentiating an acoustic neuroma from other cerebellopontine angle lesions. In: Tos M, Thomsen J, eds. Acoustic neuroma. Proceedings of the first international conference on roma. Proceedings of the first international conference on acoustic neuroma, Copenhagen, Denm

5 Luxon LM. Disorders of hearing. In: McDonald WI, Asbury AK, McKhann GM, Wansiewicz WB, eds. Diseases of the nervous system: clinical neurobiology. Philadelphia: WB Saunders, 1992, 434-50.

6 Jastreboff PJ. Phantom auditory perceptions (tinnitus). Mechanisms of generation and perception. Neurosciences 1990;8:221-54.

7 Lenarz T, Schreiner C, Snyder RL, Ernst A. Neural mechanisms of tinnitus. Eur Arch Otorhinolaryngol 1993;249: 441-6.

8 Granit R, Leksell L, Skoglund CR. Fibre interaction in injured or compressed region of nerve. Brain 1944;67: 125-40.

9 Moller AR. Pathophysiology of tinnitus. Ann Otol Rhinol Laryngol 1984;93:39-44.

10 McFadden D. Tinnitus; fact, theory and treatment. Washington: National Academy Press, 1982

11 Prasher D, Ryan S, Luxon LM. Contralateral suppression of transiently evoked oto-acoustic emissions and neurootology. Br ₹ Audiol 1994;28:247-54.

12 Jannetta PJ. Neurovascular compression in cranial nerve and systemic disease. Ann Surg 1980;192:518-25.

13 Bridger MWM, Farkashidy J. The distribution of neuralgia and Schwann cells in 8th nerve of man. $\mathcal{F}$ Laryngol Oto 1980;94:1353-62.

14 Bertrand RA, Molina P, Hardy J. Vestibular syndrome and vascular anomaly in the cerebello-pontine angle. Acta Otolaryngol 1977;83:187-94.

15 Moller MB, Moller AR, Jannetta PJ, Jho HD. Vascular decompression surgery for severe tinnitus. Laryngoscope 1993:103:421-7.

16 Isu T, Ito T, Murai H, Yamamoto K. Paroxysmal tinnitus and nystagmus accompanied by facial spasm. Surg Neurol 1985;23:183-6.

17 Kondo A, Ishikawa I, Hiwatashi S, Yamasaki T, Koyama $T$. Surgical results of the treatment of hemifacial spasm tinnitus and trigeminal neuralgia by the method of "microvascular decompression". Neurol Surg 1979;7: 677-85.

18 Jannetta PJ. Observations on the etiology of trigeminal neuralgia, hemifacial spasm, acoustic nerve dysfunction and glossopharyngeal neuralgia. Definitive microsurgical treatment and results in 117 patients. Neurochirurgia 1977;20:145-53.

19 Osterman PO, Westerberg C-E. Paroxysmal attacks in multiple sclerosis. Brain 1975;98:189-202.

20 Twomey JA, Espir MLE. Paroxysmal symptoms as the first manifestations of multiple sclerosis. $\mathcal{F}$ Neurol Neurosurg Psychiatry 1980;43:296-304. 